特集論文「Web インテリジェンスとインタラクションの新展開」

\title{
Community Detection in Scale-Free Networks using Edge Weight and Modularity Optimization Method
}

\author{
Sorn Jarukasemratana \\ Tokyo Institute of Technology \\ sorn.jarueai.cs.titech.ac.jp \\ Tsuyoshi Murata \\ (affiliation as previous author) \\ murata@cs.titech.ac.jp
}

keywords: community detection, scale-free network, edge weight, modularity

\begin{abstract}
Summary
In this paper, we propose a two-step algorithm to perform a community detection in scale-free networks. One of the main characteristics of scale-free networks is that node degree distribution follows a power law. However, during our own experiments, we encountered another sub-type of scale-free networks which we call "mixed scalefree networks". Some communities have hub nodes and node degree follows power law distribution, while some communities do not have hub nodes and node degree follows normal distribution. For mixed scale-free networks, methods that do not specifically design for scale-free will have difficulties because of the scale-free properties. At the same time, scale-free based methods will have difficulties because some communities have node degree follows normal distribution. In this research, we propose a community detection algorithm that can work on networks that contain both types of communities at the same time. Our method can handle this case correctly because our algorithm performs both scale-free and non scale-free approaches iteratively. To evaluate our method, we use NMI - Normalized Mutual Information - to measure our results on both synthetic and real-world datasets comparing with both scalefree and non scale-free community detection methods. The results show that, our method outperforms baseline methods on mixed scale-free networks and scale-free networks while performs equally on networks with normal degree distribution.
\end{abstract}

\section{Introduction}

Detecting communities in networks is useful for many real world applications such as recommendation systems for social networks or understanding the structure of the networks for biological networks. One of the important concept in community detection is modularity [Girvan 02]. Modularity is a function that measures the quality of a division of a network into groups or communities. High modularity score means there are more connections between the nodes within the same community, than connections among different communities. Since better modularity value indicates the better community detection, many community detection algorithms [Fortunato 10] aim to increase the modularity score by using modularity optimization methods. Modularity optimization based algorithms such as simulated annealing [Dorso 93], spectral optimization [Shen 10], or Louvain algorithm [Blondel 08] are known to have good community detection results especially on networks that node degree follows normal distribution. But in case of scale-free networks [Barabási
03] where node degree follows power law distribution and high degree nodes in the community are called hub nodes, these methods are not suitable. Many researches such as the one by Lancichinetti and Fortunato [Lancichinetti 09a] is confirming this fact. Therefore, many community detection algorithms for scale-free networks are proposed, such as the ones by Xu et al. [Xu 07], Qian et al. [Qian 07] or Jarukasemratana et al. [Jarukasemratana 13].

However, during our own experiments on scale-free networks, we encountered another type of network that does not fully follow the characteristics of scale-free, which we call "mixed scale-free network". Some communities have hub nodes and node degree follows power law distribution, while some communities do not have hub nodes and node degree follows normal distribution. For mixed scale-free networks, modularity optimization based methods will have difficulties because some communities contain scale-free property. At the same time, scale-free based methods will have difficulties because some communities have node degree that follows normal distribution. In this research, we propose a community detection algorithm 
that can work on networks that contain both types of communities at the same time.

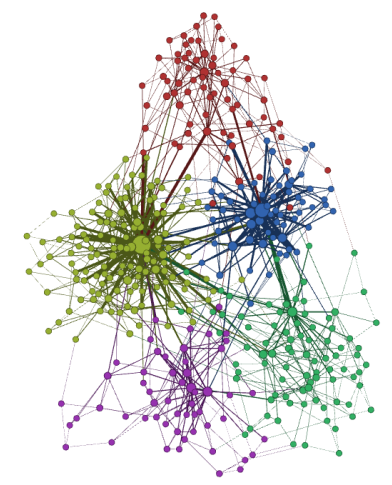

(a) Ground truth

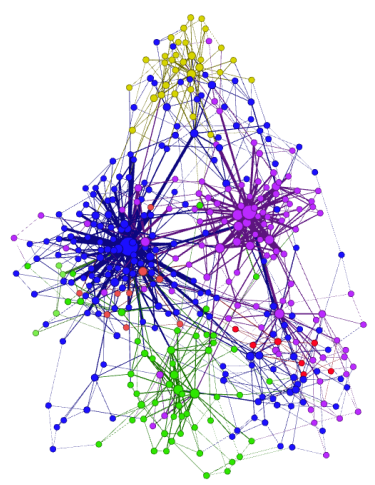

(c) Node centrality algorithm $\mathrm{NMI}=0.246$

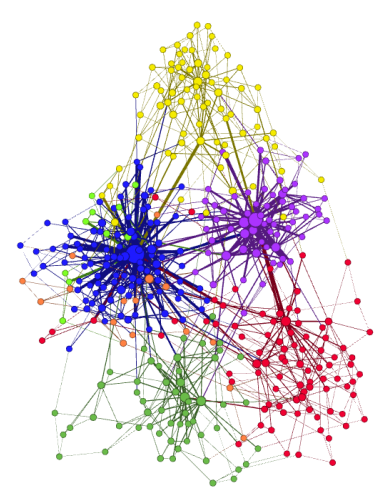

(b) Lovain algorithm, NMI $=0.595$

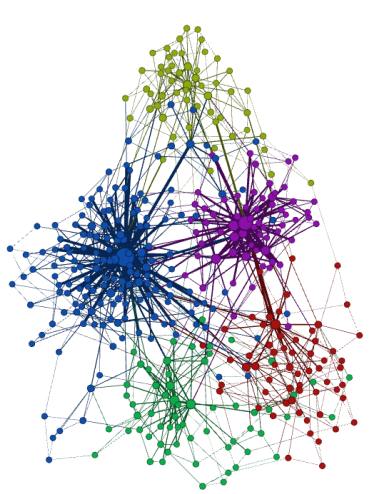

(d) Proposed algorithm $\mathrm{NMI}=0.706$
Fig. 1 Ground-truth value and community detection results of synthetic network_m2. Node size is correspondent to node degree, the bigger the node size, the higher the node degree. Higher NMI - Normalized Mutual Information - means the result is more similar to the ground-truth (better).

Figure 1 shows the result of our proposed algorithm compared with Louvain algorithm [Blondel 08] and node centrality algorithm [Jarukasemratana 13]. Figure 1(a) is the ground-truth community of the network. In Figure 1(b), Louvain algorithm failed to extract the largest community (blue color in Figure 1(b)). This community's node degree follows a power law (scale-free). The result contains 3 small communities (blue orange and green) instead of 1 large community. On the other hand, in Figure 1(c), node centrality algorithm failed to extract the bottom right community. This is because the algorithm is based on finding hub nodes in order to start the community, but there is no hub node in this community (communities that node degree follows normal distribution do not have hub nodes). What our algorithm did is to take the advantages of both approaches and to combine them into one result. We used NMI - Normalized Mutual Information - as a metric of the community detection. NMI is used for comparing the result of the community detection with the ground-truth value of the network. The result is that our algorithm received the highest NMI (Figure 1(d)) which means the result is the most similar to the ground-truth.

Mixed scale-free networks are often observed in social networks. Figure 2 is one of the network from Ego Facebook datasets [McAuley 12]. Red community on the left does not contain any hub nodes, most nodes have almost the same size (almost the same average degree), and node degree follows normal distribution. On the other hand, community on the right (blue) has high degree nodes in the middle surrounded by lesser degree nodes, and node degree of the community follows a power law, which is similar to scale-free networks.

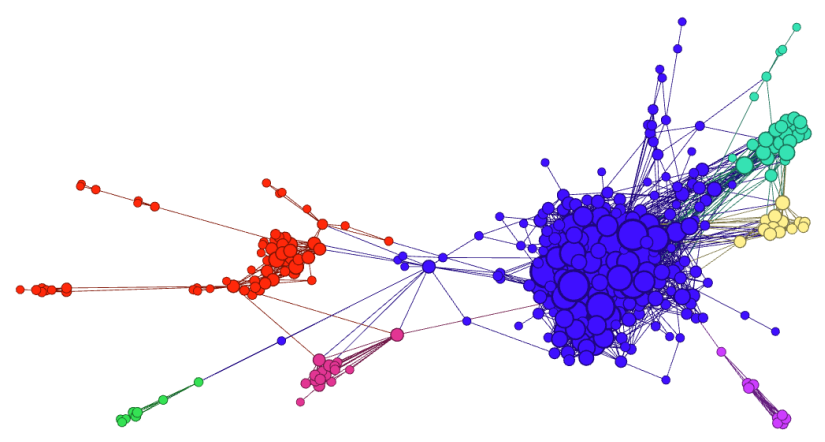

Fig. 2 Facebook0 network from Ego Facebook datasets [McAuley 12], the example of networks that contain both communities with normal distribution and power law distribution, red community's node degree follows normal distribution, while blue community's node degree follows power-law distribution.

The paper is organized as follows. In section 2, related works are reviewed. In section 3 , our algorithm is explained. In section 4, we show our algorithm results compared with modularity optimization based algorithm, scale-free algorithm, betweenness centrality algorithm, and label propagation algorithm. In section 5, the results from section 4 are discussed. The last section is the conclusion.

\section{Related Work}

Our algorithm consists of many steps and contains many ideas based from previous works in this field. This section is dedicated to those previous works. First of all, in the first step of our algorithm, we have to calculate the weight of each edge. This concept is similar to the famous work by Girvan and Newman's edge betweenness algorithm [Newman 03]. Edge betweenness value is calculated based on the number of the shortest paths that go through that edge. Normally, betweenness value of edges that connects communities together is high. In GirvanNewman algorithm, betweenness of all edges are calculated. After that, the edge with the highest betweenness 
value is removed. Finally, the betweenness is calculated again followed by another edge removal. This process is repeated until no edges left. The difference from our algorithm is that our weight is not directly coincide with the betweenness, but is a mixture of centrality and local clustering coefficient. Moreover, Girvan-Newman is a top down approach, which treats all nodes as one community first, then divides them up into several communities. Our algorithm, instead, is a bottom up approach. Each node has its own community at first, then the algorithm combines them into bigger communities.

There are many community detection methods that aim for scale-free networks. One of them is a method based on node centrality and edge distance [Jarukasemratana 13]. The method starts with the calculation of node centrality and edge distance. Node centrality is used to find hub nodes and edge distance is used for determining the communities. The largest difference from our proposed algorithm and node centrality algorithm is that our proposed algorithm is focused on edges instead of nodes. By using edge weight, scale-free communities can be extracted while allows communities with normal distribution to be extracted in the latter step. Node centrality algorithm also fails to extract communities that do not have hub nodes, which normally appear in mixed scale-free networks.

The latter part of our algorithm is modularity optimization based method. Since our method is a bottom up approach, it is similar to the second iteration of Louvain algorithm [Blondel 08]. In Louvain algorithm, first, each node is randomly selected and paired with other node in order to create the highest modularity gain pair. Then, the current network is transformed into a new network where nodes are the communities of the current network. After that, the first and the second steps are repeated until the maximum modularity is gained. Therefore, in our algorithm, the starting network for Louvain algorithm is a processed network created by our scale-free part. Another difference from normal Louvain algorithm is that edges in our algorithm have weights and the calculation order is based on weight which makes our algorithm deterministic.

To measure the result of the community detection, we use NMI [Lancichinetti 09b]. NMI is used to determine the similarity between two partitioning results. In order to obtain the accuracy of the community detection result, we have to calculate the NMI between the ground-truth communities and detected communities. The higher the score is the closer the result is to the ground-truth. In many real-world networks, there is no absolute truth value. Thus using NMI is limited to the well-known networks whose truth values are available or synthetic networks where truth

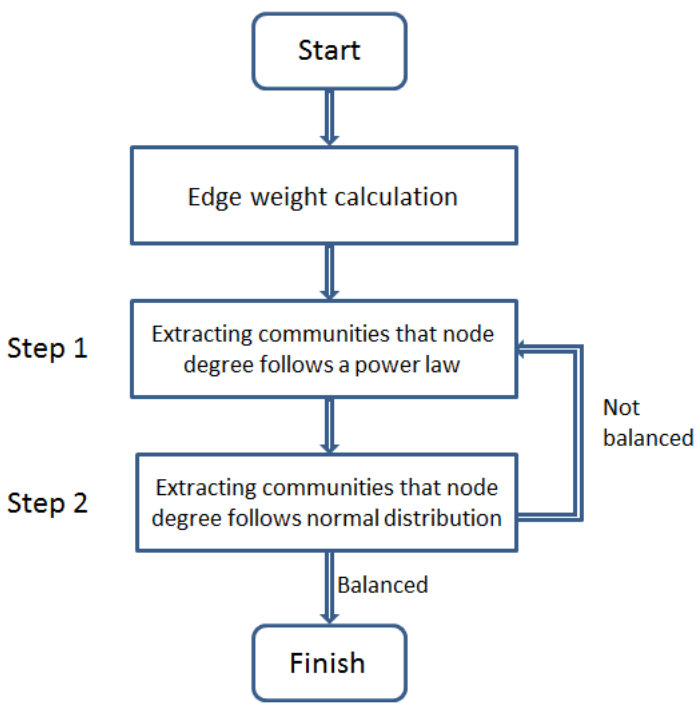

Fig. 3 Flowchart of proposed algorithm.

values are already predetermined. There are many versions of NMI algorithms available. The main difference among NMI algorithms is the ability to give an appropriate score for overlapping communities. However, our algorithm generates disjoint communities. In this research, we use the version by Lancichinetti, Fortunato and Kertész [Lancichinetti 09b] because it is shown in their research that their NMI values can perform on disjoint communities similar to other NMI values. The synthetic networks generated in this research are created according to "Benchmark graphs for testing community detection algorithms" by Lancichinetti, Fortunato, and Radicchi [Lancichinetti 08].

\section{Our Algorithm}

We believe that edges are what determine the communities of the network. Also, each edge is not equal. Therefore, the main idea of our algorithm is to weight each edge according to its importance, then community detection is performed based on the weighted network. The summary of our algorithm is explained here. First of all, weight of each edge is calculated by using centrality method with attenuation factor along with local clustering coefficient. Once we have all edges weighted, community detection phase is performed. Since we have two types of communities (node degree follows a power law and node degree follows normal distribution), we performs two community detection algorithms for each type of community. In step 1 , the first algorithm is aimed for extracting communities that node degree follows a power law. In step 2, the second algorithm is aimed for extracting communities that 


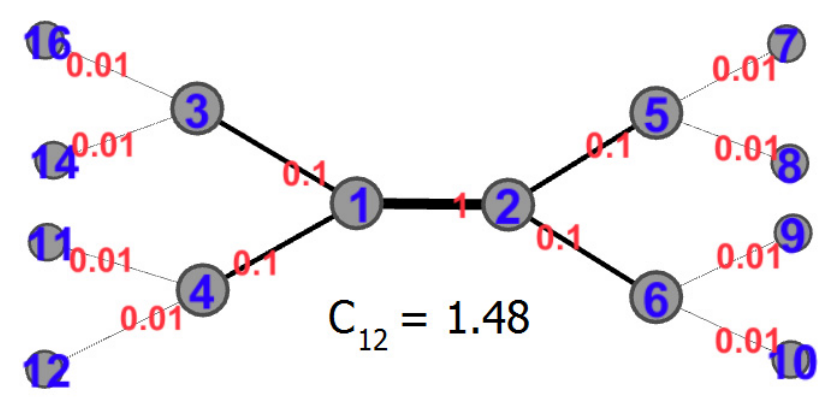

Fig. 4 The example of edge weight calculation, our target is the weight of an edge that connecting node 1 and 2 . Let attenuation factor $\alpha$ is set to 0.1 . An edge itself weight 1.0. There are 4 edges that are located 1 hop away from this edge, resulting in $\alpha$ times $4=$ 0.4 . There are 8 edges that are located 2 hops away, resulting in $\alpha^{2}$ times $8=0.08$. Therefore, using $l=2$, This edge's weight is $1+0.4+0.08=1.48$.

node degree follows normal distribution. Step 1 and step 2 are performed iteratively until the result is balanced or the number of iteration threshold is met. The whole process is explained with a flow chart in Figure 3.

\subsection{Edge Weights Calculation Phase}

The purpose of calculating edge weight is to determine whether the nodes that are linked by this edge should be in the same community or not. If the weight is high, it means that nodes that are connected by this edge should be in the same community. Therefore, the weight of edges that connect nodes inside same community should be high. On the other hand, if the weight is low, these two nodes should not be in the same community. Edges that connect nodes from different communities should have low weight. To explain with metaphor, edges in our algorithm are similar to rubber bands that connecting nodes together. Edge weight represents the thickness of the rubber bands. The higher the weight, the more pulling power between connected nodes. To calculate this weight, we apply the node centrality method base on Katz centrality [Foster 01], but we use it on edges instead:

$$
C_{i j}=\sum_{l=0}^{n} \alpha^{l} E_{l},
$$

where $C_{i j}$ is the centrality of an edge that connects node $i$ and node $j, \alpha$ is the attenuation factor of Katz centrality, $n$ is the maximum depth, $E_{l}$ is the number of edges that are located $l$ hops away. If the network is weighted, $E_{l}$ is equal to the sum of all weight that are located $l$ hops away. An attenuation factor is used for reducing the score that generated from nodes that are located $l$ hops away. Figure 4 shows an example on how to calculate $C_{i j}$. If the edge connects nodes with high degree, the centrality of that edge is high. This centrality value can separate edges that connect to important nodes (such as hub nodes in scalefree networks) from edges that connect to less important nodes. However, in some cases, the centrality value alone is not enough. For example, edges that connected two hub nodes from different communities normally have very high centrality, which results in a merging of two communities. To prevent this from happening, we added local clustering coefficient into edge weight. Local clustering coefficient can differentiate edges inside the same community from edges that link communities together. Edges that bridge two communities together normally have low local clustering coefficient. Local clustering coefficient can be counted by the number of triplets ( 3 nodes and 3 edges forming a triangle shape, a 3-clique). For each triplet that the edge participated in the weight is increased by 1 (in case of unweighted networks). For weighted networks, the weight is increased by its original weight. The number of triplets can be calculated by using the power of 3 of the adjacency matrix. The last step is to normalize the value, for each edge weight, we divide it by the average value of all edge weight. This process is needed because in some extreme case, the difference in edge weight is very high. For example, in a very dense area of the graph, the weight of each edge is extremely high. When community detection phase is performed, communities from sparser area will be merged into communities from denser area. The final step is to add our normalized weight to current edge weight. In case of unweighted graphs, each edge weight is one. The final formula is shown as follows:

$$
W_{i}=w_{i}+\left(C_{i}+T_{i}\right) /(\bar{C}+\bar{T})
$$

where $w_{i}$ is the original weight of edge $i, C_{i}$ is the centrality of edge $i, T_{i}$ is the number of triplets that edge $i$ participated in, $\bar{C}$ is the average value of edges centrality, and $\bar{T}$ is the average number of triplets that edges participated in. If the network is weighted, $w_{i}$ is equal to the original weight of edge $i$. In case of unweighted edges, $w_{i}$ is equal to 1 . From our experiments on unweighted networks, edge weight normally ranges from 1 to 4 in case of scale-free networks. The higher values are from edges that connect to hub nodes. Also, the number of edges that has weight less than 2 is significantly larger than the number of edges that weight more than 2 . This is according to the structure of scale-free networks that node degree follows power law distribution. On the other hand, in networks that node degree follows normal distribution, most weight values are around 2. An example of edge weight from real dataset can be seen in Figure 5. It can be seen that edges that connect between communities are thin (low 


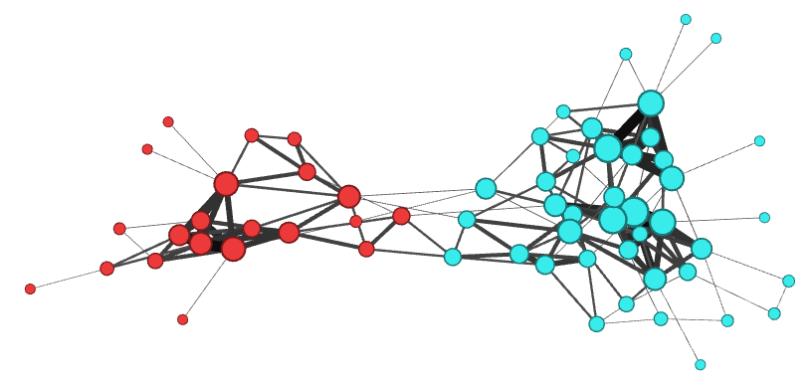

Fig. 5 Doubfulsound dolphins network [Lusseau 03] after edge weights calculation. Node's size refers to node's degree, the bigger the higher. Edge's thickness refers to its weight, the thicker edges weight more than the thinner edges. It can be noticed that edges that connected to high degree nodes (hubs) have considerably higher weight. Moreover, communities are divided where edge weight values are low (thin edges).

weight), while edges in the center of each community are thick (high weight).

\section{$3 \cdot 2$ Community Detection Phase}

There are 2 steps in community detection phase. The first step is aimed to extract communities with power law distribution (scale-free), while the second step is aimed for communities with normal distribution.

\section{$\S 1$ Step 1}

Since high weight edge means nodes that are connected by this edge should be in the same community, edges with higher weight should be processes first. This step starts with sorting all edges from the highest weight to the lowest weight and assigning each node a unique community. Step 1 algorithm is starting from the pair of nodes that are connected by the highest weight edge. In this pair of nodes, the node which have higher sum of edge weight is processed first. This makes our algorithm deterministic which results in a unique solution.

For each node, we create a list of edges that connect to it. From this list, weights of the edges that connect to the node with the same community are summed together. For example, in Figure 6, node $a$ connects to two communities, red and blue. The sum of weight from blue community is 2.4 while the sum from red community is 1.3 . If the highest weight community is not this node's current community, the node will change community to the community with the highest total weight instead (this node got pulled to the highest weight community). As in Figure 6 , node $a$ is going to change community from red to blue because the sum of the weight from blue community is higher than the sum of the weight from red community. This process is repeated iteratively until all nodes are balanced or until the threshold is met. The pseudo code of

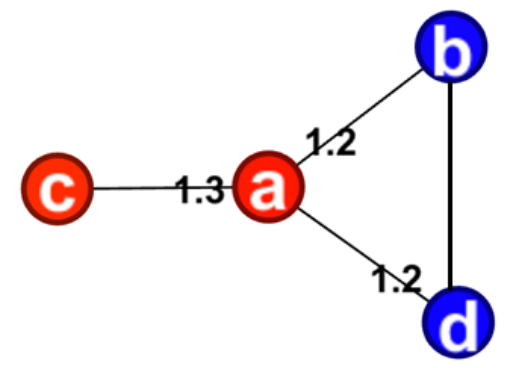

Fig. 6 Node $a$ is going to change from red community to blue community, because the total weight from blue community is 2.4 (two 1.2 from node $c$ and node $d$ ) while total weight from red community is 1.3 (from node $b$ ).

this step is shown in Algorithm 1.

The communities that follow scale-free are extracted almost completely in this step. This is because in scale-free community, edges that connected with hub node tend to have very high weight. Edges around hub nodes will be processed first. Figure 5 is an example of edge weight of our algorithm. It can be seen that edges in the center of the communities have higher weight than edges at the peripheral area of the communities. Therefore, if this process is observed step by step, it can be noticed that the community is extracted starting around nodes in the middle of communities where edge weight is high and then expanding to the peripheral area of each community. On the other hand, in communities with normal distribution, each edge weight is almost the same. Therefore, nodes in communities with normal distribution are randomly grouped up into small sub-communities without any significant meaning. An example result of step 1 is shown in Figure 7.

node.sort(HighestEdgeWeight);

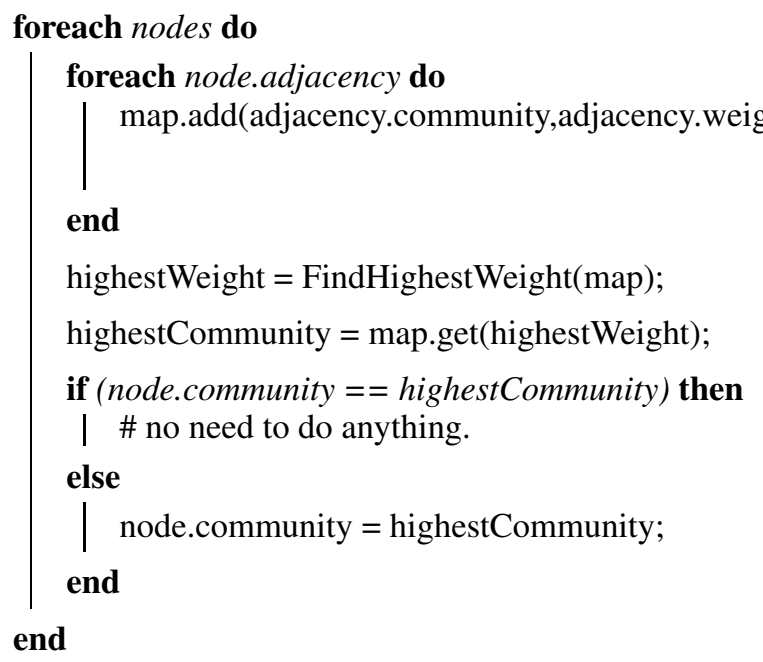

Algorithm 1: Community Detection Phase - Step 1 


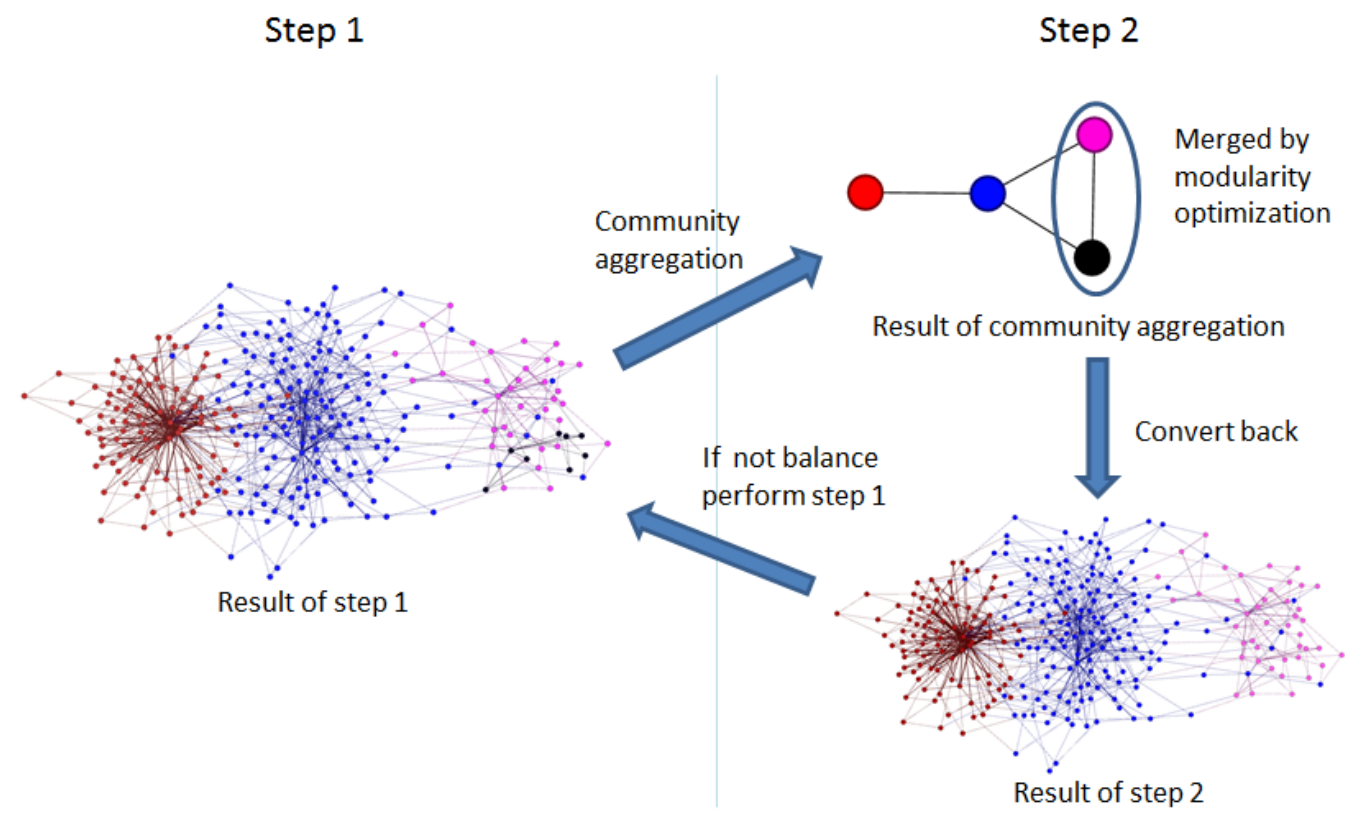

Fig. 7 An example of the iteration loop of step 1 and step 2. In the result of step 1, left (red) and middle (blue) communities are already extracted, but right communities still got separated into two communities (pink and black). Step 2 begins with community aggregation resulting in a network with only 4 nodes. Modularity optimization is performed in this aggregated network, resulting in the merge of black and pink communitues. At the end of step 2 , the graph is converted back to its original state. Step 1 is performed again if the result is not balanced.

\section{$\S 2$ Step 2}

The goal of this step is to extract communities that node degree follows normal distribution. It has been proved that modularity optimization based method is excellent at this task. Therefore, in this step, we applied the modified version of one of the most popular modularity optimization algorithm, Louvain algorithm [Blondel 08]. In normal Louvain algorithm, the first step is to randomly select nodes and try to pair it with other nodes in order to obtain sub-communities of the highest modularity. When all nodes are processed, this step is done. The second step is to aggregate communities generated in the first step into a node. With this method, the network obtained in this step is much smaller from the original network. This is the end of the first iteration. The first and the second step are repeated iteratively until the maximum modularity is gained. In our case, our previous steps are equal to the first step of Louvain algorithm. In step 2 of our algorithm, we start with the aggregation of our current network into a new network where each community is represented as a node. After that, starting from the highest total weight node, modularity optimization is applied until the maximum modularity of the whole network is obtained. Since the random part of original Louvain algorithm is replaced, this makes step 2 deterministic and gives a unique result. Moreover, using our weighting scheme for modularity optimization results in a better NMI than non-weighted version. This is further explained in discussion section.
After step 2 is done, if there are merges of communities in step 2, it is possible that some nodes at the peripherals of communities should be change to other communities from the effect of step 1. Therefore, if the change occurs in step 2, we have to perform step 1 again. And if there is any change in step 1 , step 2 is needed to be performed again as well. The algorithm is finished when both step 1 and step 2 are at balanced state (nodes no longer change communities in both steps) or the threshold is met. The example of iteration between step 1 and step 2 is shown in Figure 7.

\section{$\S 3$ Configurable Parameters}

There are two configurable parameters in this algorithm, attenuation factor $\alpha$ and the depth $l$. Both are used when calculating edge weight. The term attenuation factor is used in Katz centrality [Foster 01]. Attenuation factor is used for penalizing the connections that are farther away. This value should be set to range from 1 to 0 . Setting this value more than 1 will result in farther away edges are more important than the edge itself (switch from penalizing to rewarding edges for locating farther away). In our algorithm, $\alpha=0.1$ is employed. This value is selected because, from our experiments, it gave the most balanced result from both normal degree distribution graphs and scale-free graphs from our experiments. Another configurable value is depth $l$. The higher the depth is, the more accurate the weighting scheme is. In our case, the minimum $l$ is 2 . This is because we need three edges 


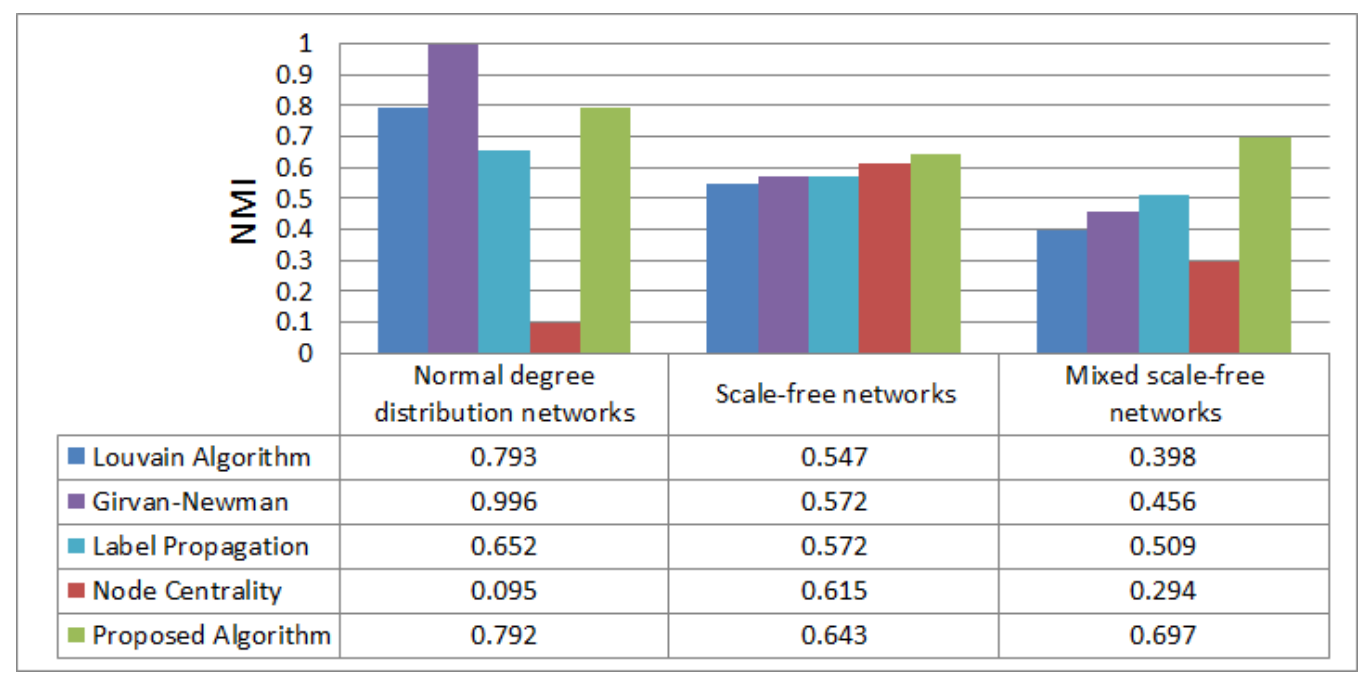

Fig. 8 NMI results on normal degree distribution networks, scale-free networks, and mixed scale-free networks.

to form the triplets to calculate the clustering coefficient. From our observation, $l=2$ (minimum value) provides the best trade-off point for calculation time and performance. However, in small networks, we used $l=3$ in our experiments to increase the accuracy.

\section{Experiments}

Since we used NMI as our benchmark, networks used in the experiments are required to have ground-truth. This severely limits our choices for real-world networks. To expand our choices, we used "Benchmark graphs for testing community detection algorithms" [Lancichinetti 08] to create synthetic networks with ground-truth value. NMI is used as a metric of community detection. The higher the NMI is, the closer the result of community detection is to the ground-truth. The maximum NMI score is 1.0 which means the result is exactly the same with the ground-truth. All figures of networks in this research is displayed by Gephi [Bastian 09] using Yihan Fu and ForceAtlas 2 layout algorithm.

The first experiment is to test our algorithm on three categories of synthetic networks; networks with normal distribution, networks with power law distribution (scalefree networks), and networks that some communities have node degree follows a power law while some communities have node degree follows normal distribution (mixed scale-free networks). We compared our proposed algorithm with modularity optimization based algorithm (Louvain algorithm), scale-free based algorithm (node centrality algorithm), Girvan-Newman edge betweenness algorithm [Newman 03], and label propagation algorithm [Raghavan 07]. Mixing parameter $\mu$ of synthetics networks in this experiment is set to 0.2. Mixing parameter is equal to noises in networks, the higher the value, the more edges that connect between two communities. For node centrality algorithm, laxing value - percentage value that use for combining nodes into sub-communities - is set at $70 \%$ for normal distribution and $90 \%$ for scale-free. The results are shown in Figure 8. It can be seen that our method has about the same NMI as modularity optimization based method on normal distribution networks, slightly better NMI than baseline algorithms on power law distribution networks, and clearly outperformed all baseline methods on mixed scale-free networks. Node centrality algorithm, which focuses solely on the characteristic of scale-free networks such as hub nodes, can make mistakes when some communities do not follow scale-free characteristic. The example of this phenomenon is shown in Figure 1. Girvan-Newman edge betweenness algorithm received the highest score on normal distribution networks. However, the most disadvantage of edge betweenness algorithm is the time complexity. Because the algorithm needs to recalculate all the centrality values in each iteration, this algorithm is only suitable for small size networks. Another possibility is that this network synthesizer is overfitting with this algorithm. On the other hand, label propagation algorithm is extremely fast. The authors claimed that the complexity is almost linear [Raghavan 07]. However, the result of label propagation is in the middle on all types of networks.

The second experiment is to test our algorithm on realworld datasets that have ground-truth value. Real-world networks used in this experiments are Zachary karate club network [Zachary 77], Doubtfulsound dolphins network [Lusseau 03], American college football network [Girvan 02], and Political books network [Girvan 02]. The result is shown in Table 1, Figure 9, and Figure 10. In this exper- 
Table 1 The NMI results of our proposed algorithm compared with other algorithms

\begin{tabular}{|c|c|c|c|c|c|}
\hline NetworkName & Louvains & Girvan - Newman & LabelPropagation & NodeCentrality & ProposedAlgorithm \\
\hline Zachary karate club & 0.359 & 0.370 & 0.678 & 1.0 & 1.0 \\
American College Football & 0.765 & 0.723 & 0.725 & 0.765 & 0.765 \\
Doubtfulsound dolphins & 0.328 & 0.486 & 0.332 & 0.609 & 0.580 \\
Political books & 0.349 & 0.318 & 0.238 & 0.308 & 0.449 \\
\hline
\end{tabular}
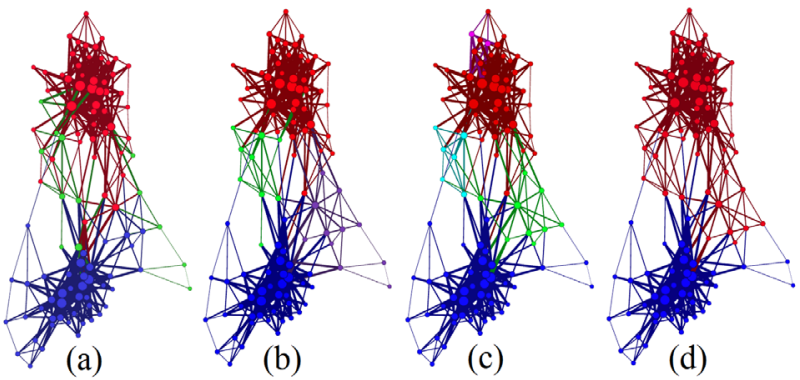

Fig. 9 Political books network; (a) Ground-truth , (b) Louvain algorithm - NMI $=0.349$, (c) Node centrality $-\mathrm{NMI}=0.308$, (d) Proposed method $-\mathrm{NMI}=0.449$

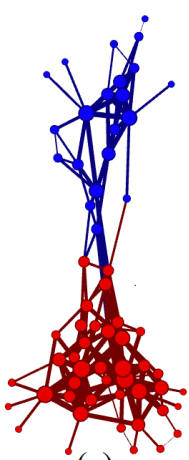

(a)

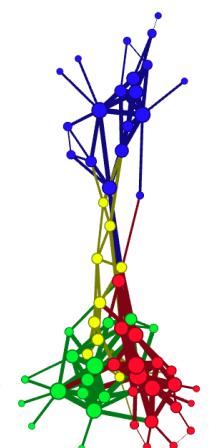

(b)

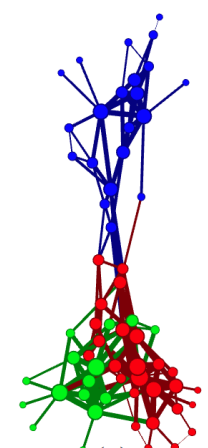

(c)

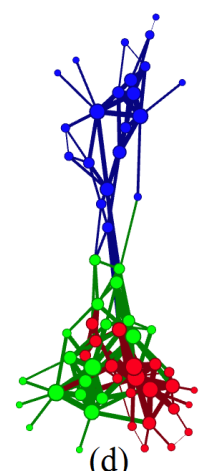

(d)
Fig. 10 Doubtfulsound dolphins network; (a) Ground-truth , (b) Louvain algorithm - NMI $=0.328$, (c) Node centrality $-\mathrm{NMI}=$ $0.609,(\mathrm{~d})$ Proposed method $-\mathrm{NMI}=0.580$

iment, Girvan-Newman edge betweenness algorithm did not receive the highest NMI scores in any networks, which may ensure that the synthetic networks used in the first experiment are overfitting with edge betweenness algorithm.

The third experiment is to test our algorithm on synthetic scale-free networks but the mixing value is set from 0.1 to 0.4 by increasing mixing value by 0.5 per step. The result is shown in Figure 11. It can be seen that our proposed algorithm starts to fall off at mixing value 0.25 . After that, both algorithms cannot maintain solid community detection results anymore.

The last experiment is to apply our algorithm on realworld networks that do not have ground-truth value. Ego Facebook networks [McAuley 12] are used in this experiment. Since NMI requires ground-truth value, it is not possible to measure the correctness of the community de-

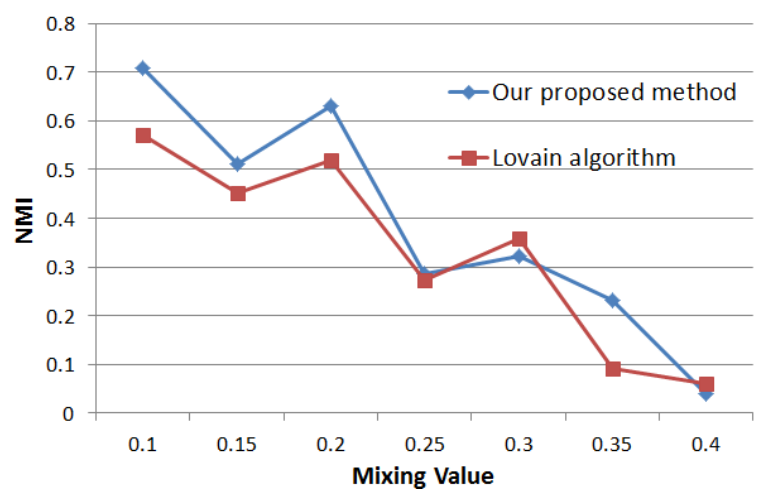

Fig. 11 Results from the third experiment, mixing value is equal to noises in networks.

tection results. However, some networks exhibited the same results similar to when we performed community detection on mixed scale-free networks. These results are shown in Figure 13 in discussion section.

\section{Discussion}

\section{$5 \cdot 1$ Experimental Results}

For the first experiment, the first type of networks is the networks with normal distribution. Our algorithm achieves about the same NMI with modularity optimization method, receives better NMI when compared with scale-free and label propagation methods, and performed worse when compared with Girvan-Newman edge betweenness method. Next type of networks is networks with power law distribution or scale-free networks. Our algorithm achieves slightly better NMI over baseline methods. However, on mixed scale-free networks, our proposed algorithm outperformed all other methods. The result is as expected because our method is the combination of scale-free based and modularity optimization based method. The analysis of the result on mixed scale-free is shown in Figure 12.

For method that aimed for networks that node degree follows normal degree distribution, it tends to break large communities that node degree follows a power law (Figure 12 (a), red circle). This is because small groups of nodes formed a tightly connected group inside the community, which makes large community breaks into several smaller communities. Methods that are designed for scale- 


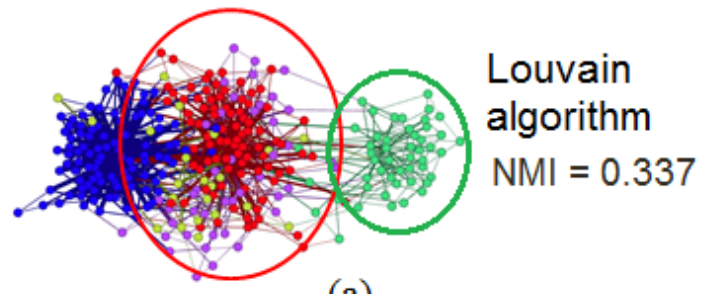

(a)

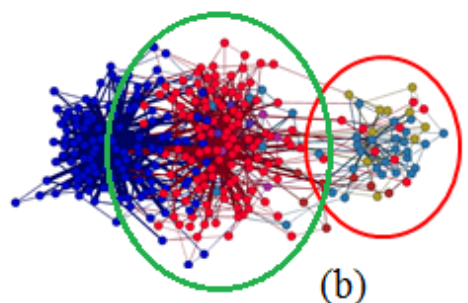

Node centrality algorithm

$\mathrm{NMI}=0.287$

(b)

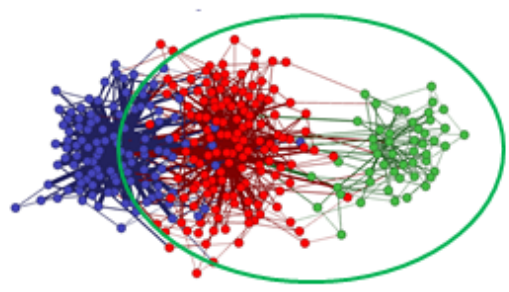

Proposed algorithm

$\mathrm{NMI}=0.836$

(c)

Fig. 12 Community detection results on a mixed scale-free network; (a) Louvain algorithm, (b) Node centrality algorithm, (c) Proposed algorithm.

free can detect this type of community correctly because it can detect the hub nodes of the community (Figure 12 (b), green circle). On the other hand, methods that is designed for scale-free cannot detect communities that node degree follows normal distribution because these communities do not have hub nodes (Figure 12 (b), red circle), while methods that aimed for networks that node degree follows normal degree distribution can identify these communities correctly (Figure 12 (a), green circle). However, our proposed method is designed to bring the correct parts of both types of community detection methods. Therefore, our method can correctly extract both types of communities and achieved the highest NMI (Figure 12 (c)).

Figure 13 is the results of one of the Ego Facebook datasets from 3 community detection algorithms, Louvain algorithm, node centrality algorithm, and proposed algorithm. It can be seen that in Figure 13 (a), which is the result from Louvain algorithm, the group of nodes in the red circle is broken into several communities. This is very similar to what occurred in Figure 12 (a). In Figure 13 (b), which is the result from node centrality algorithm, the group of nodes in the red circle is also broken into several communities, similar to what occurred in Figure 12 (b). Figure 13 (c) is the result of our proposed algorithm,

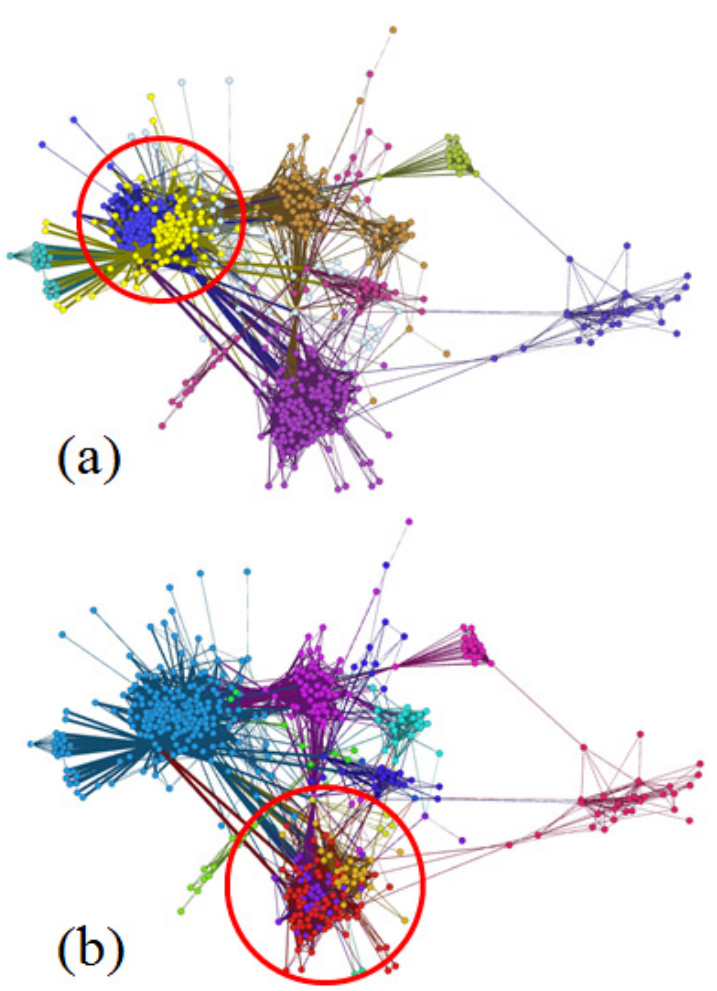

(c)

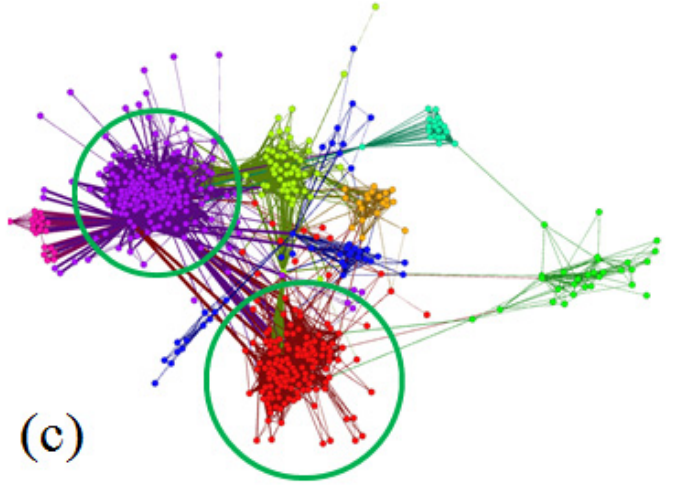

Fig. 13 Community detection results on Facebook0 network. (a) Louvain algorithm, (b) Node centrality algorithm , (c) Proposed algorithm.

all the largely connected group can be extracted without broken into several communities.

The largest disadvantage of our algorithm is the vulnerability against noises. As shown in the third experiment, our algorithm is tested on scale-free networks where mixing parameters are ranging from 0.1 to 0.4 . It can be seen that the average NMI of our algorithm drops very fast. Figure 14 shows the result of our proposed algorithm compared with ground-truth and Louvain algorithm on a synthetic network. It can be seen that our algorithm considered the whole graph as one huge community, while the truth is not. The reason is because in the first step of our community detection phase, we merge nodes together from the highest edge weight to the lowest edge weight. In case of low noise situations, edges that connect between 
communities normally have low weight. These edges are processed in the latter part of the algorithm where most communities are already formed up. However, when there are a lot of noises, it is possible that these noise edges will have high weight. This results in the merging of communities that these noise edges connected to. In the worst case such as shown in Figure 14, the whole graph is merged into one large community. This phenomenon does not happen in modularity optimization based algorithm because before merging of communities, the modularity must be calculated first, which means it is not possible for all communities to merge into one large community. We can use this idea to improve our algorithm in the further researches.

\subsection{Time Complexity}

Another aspect of the algorithm that needs to be mentioned is the complexity or computational time. The tradeoff for combining two algorithms into one is that our method has many steps, which is costly. In edge weight phase, the complexity is $O\left(m k^{l}\right)$, where $m$ is the number of edges, $k$ is the average degree, and $l$ is the depth of the algorithm. Minimum value of $l$ is 2 . It should be noted that in this phase, if the number of edges is the same, networks with more nodes are faster. This means that our algorithm is suited for sparse networks than dense networks. The first step in community detection requires a run through all nodes once for each iteration. The number of iteration required is unknown, but from our experiments the number of iteration never exceeds 10 . Thus complexity in this step is $O(n k)$, where $k$ is the average degree and $n$ is the number of nodes. The second step has the complexity of Louvain algorithm with slight difference because the number of nodes is the number of communities aggregated from previous steps. So, the complexity of this step is $O(n \log n)$, where $n$ is the number of communities aggregated from previous steps, which is normally small. Based on our test on various networks, in most case, more than $70 \%$ of the time is spent on edge weight phase. Therefore, we like to assume that the complexity of our algorithm follows the complexity of edge weight step, which is $O\left(m k^{l}\right)$, where $m$ is number of edges, $k$ is average degree, and $l$ is the depth of the algorithm.

We test our algorithm on real-world datasets of various sizes to measure our algorithm computational time. The networks used are Arxiv General Relativity and Quantum Cosmology collaboration network (GRQC) [Leskovec 07], Gnutella peer to peer network 8 [Ripeanu 02], Arxiv high energy physics theory citation graph (HepTh) [Leskovec 05], Pretty Good Privacy network (PGP) [Boguñá 04], and
DBLP collaboration network [Yang 12]. The size and the computational time of each network are shown in Table 2.

\section{$5 \cdot 3$ Weighted Modularity}

Since NMI is usable only when ground-truth is available, this makes using NMI with real world datasets almost impossible. For example, in Facebook datasets, there is no ground-truth. When ground-truth is not available, usually, modularity [Girvan 02] is used to determine the goodness of the community detection results. However, it is shown that modularity is not suitable for scale-free networks [Jarukasemratana 13]. To come up with better evaluation score, we tried to improve modularity with our edge weight technique. Modularity can be calculated by this formula:

$$
Q=\sum_{i=1}^{c} e_{i i}-a_{i}^{2}
$$

where $c$ is the number of communities, $e_{i i}$ is the sum of cell values of adjacency matrix where starting and ending nodes are both in community $i$ divided by 2 times of number of edges, and $a_{i}$ is the sum of cell values of adjacency matrix where starting or ending node are in community $i$ divided by 2 times of number of edges. In unweighted networks, values in adjacency matrix are either 0 or 1 depending on whether there is an edge between corresponding nodes or not. However, when applying our edge weighting scheme, the value in each cell is modified to match with the centrality of each edges. By using these modified adjacency matrix, we can calculate the new modularity value.

We performed an experiment to compare the NMI results of modularity, weighted modularity, and our proposed algorithm. Community detection results of modularity and weighted modularity are obtained by using Louvain algorithm [Blondel 08]. The results are shown in Table 3.

From Table 3, it can be seen that weighted modularity yields better NMI results than normal modularity. However, our proposed method still yields the best NMI results. The benefits of weighted modularity over our proposed algorithm are that the ground-truth is not required and the time complexity is better. The complexity of Louvain algorithm is $O(n \log n)$. The complexity for edge weight calculation is $O\left(m k^{l}\right)$, where $m$ is the number of edges, $k$ is the average degree, and $l$ is the depth of the algorithm. In case of extremely large networks, where our proposed method is not feasible, it is possible to use weighted modularity for a trade-off between accuracy and computation time. It is also possible to tweaking the edge weight scheme so that when maximizing the weighted modularity, the NMI results are better. Although this approach 


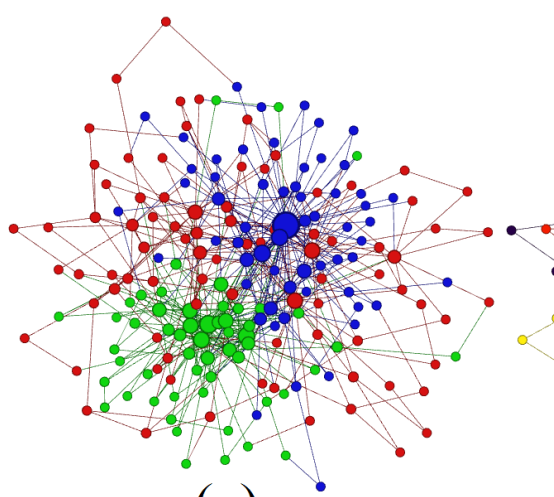

(a)

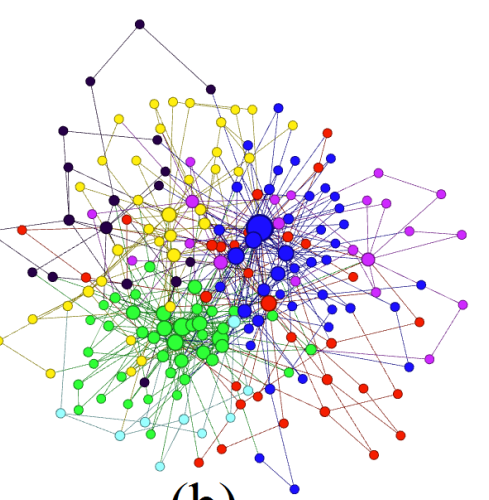

(b)

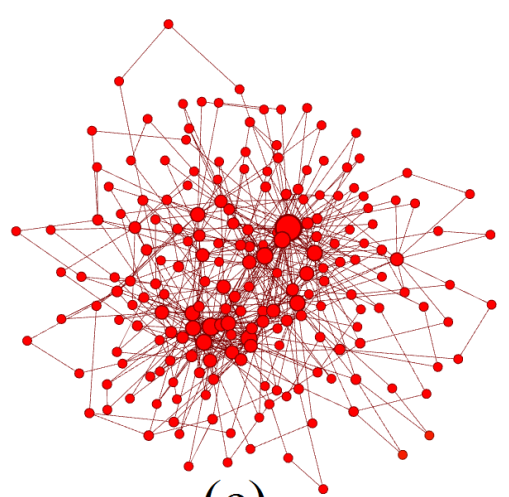

(c)

Fig. 14 Synthetic network_m3, mixing value $=0.25$ (a) Ground-truth , (b) Louvain algorithm - NMI $=0.254$, (c) Proposed method - NMI $=0.0$

Table 2 Number of nodes, number of edges, and computational time (seconds) on 5 benchmark networks

\begin{tabular}{|c|cc|cc|c|}
\hline Networks & Nunberof Nodes & NumberofEdges & TimeforWeightingStep & TimeforCommunityDetection & TotalTime \\
\hline GRQC & 4158 & 13422 & 24 & 3 & 27 \\
HepTh & 6301 & 20777 & 26 & 1 & 27 \\
Gnutella8 & 8638 & 24806 & 19 & 12 & 31 \\
PGP & 10,680 & 24,316 & 43 & 12 & 55 \\
DBLP & 317,080 & $1,049,866$ & 3029 & 3161 & 6190 \\
\hline
\end{tabular}

is not what we focused on in this research, it is very possible for us to investigate this approach in the near future.

\section{Conclusions}

In this research, we propose an algorithm for community detection that specifically aims at scale-free networks which contain both communities that node degree follows normal distribution and communities that node degree follows power law distribution, which we call "mixed scalefree network". When this algorithm is applied to networks with normal distribution, the result will be similar to those from modularity optimization based algorithms. If this algorithm is applied to scale-free networks where communities have node degree follows a power law, the result will be similar to those from scale-free based methods. And if this algorithm is applied to mixed scale-free networks, the result will be superior when compared with other methods. The key concept is that, we perform scalefree based community detection method to extract scale- free communities, then perform modularity optimization based method to extract normal distribution communities. We performed experiments to test our method by comparing the results with modularity optimization based, scalefree based, edge betweenness, and label propagation methods. We used famous real-world datasets which have groundtruth available and synthetic datasets. NMI is used for evaluation. Our method performs equally to the previous approaches on networks with normal distribution. On scale-free networks, especially on mixed scale-free networks, our method is superior. So, if the type of the networks is unknown, our method should be used because the result is equal or better than previous methods. The disadvantage of our approach is the vulnerability to noises which can result as a whole network becomes one large community. Our further research is to make this algorithm more robust to noises and receive better accuracy and computational time.

Table 3 The NMI results of modularity, weighted modularity, and our proposed algorithm.

\begin{tabular}{|c|c|c|c|}
\hline NetworkName & Modularity & WeightedModularity & ProposedAlgorithm \\
\hline Zachary karate club & 0.359 & 0.432 & 1 \\
American College Football & 0.765 & 0.765 & 0.765 \\
Doubtfulsound dolphins & 0.328 & 0.412 & 0.580 \\
Political books & 0.349 & 0.356 & 0.449 \\
\hline
\end{tabular}




\section{$\diamond$ References $\diamond$}

[Abou-Rjeili 06] A. Abou-Rjeili and G. Karypis: Multilevel algorithms for partitioning power-law graphs. In Proceedings of the 20th international conference on Parallel and distributed processing, IPDPS'06, pp. 124-124, Washington, DC, USA, (2006) IEEE Computer Society.

[Barabási 03] A.-L. L. Barabási and E. Bonabeau : Scalefree networks. Scientific American, Vol. 288 , No. 5, pp. 60-69, May (2003)

[Bastian 09] M. Bastian, S. Heymann, and M. Jacomy. Gephi: An Open Source Software for Exploring and Manipulating Networks. International AAAI Conference on Weblogs and Social Media, (2009)

[Blondel 08] V. D. Blondel, J.-L. Guillaume, R. Lambiotte, and E. Lefebvre: Fast unfolding of communities in large networks. Journal of Statistical Mechanics: Theory and Experiment, July (2008).

[Boguñá 04] M. Boguñá, R. Pastor-Satorras, A. Díza-Guilera, and A. Arenas. Models of social networks based on social distance attachment. Phys. Rev. E, Vol. 70, Issue 5, pp. 056122, Nov.(2004)

[Bonacich 07] P. Bonacich. Some unique properties of eigenvector centrality. Social Networks, Vol. 29, No. 4, pp. 555-564, Oct. (2007)

[Borgatti 05] S. Borgatti. Centrality and network flow. Social Networks, Vol. 27, No. 1, pp. 55-71, Jan. (2005)

[Dorso 93] C. Dorso and J. Randrup : Early recognition of clusters in molecular dynamics. Physics Letters B, Vol. 301, No. 4, pp. 328333, (1993)

[Estrada 05] E. Estrada and J. A. Rodríguez-Velázquez. Subgraph centrality in complex networks. Phys Rev E Stat Nonlin Soft Matter Phys, Vol. 71, No. 5 Pt 2, May (2005)

[Estrada 11] E. Estrada. The Structure of Complex Networks: Theory and Applications: Theory and Applications. OUP Oxford, (2011)

[Fortunato 10] S. Fortunato. Community detection in graphs. Physics Reports, Vol. 486, pp. 75-174, Jan. (2010)

[Fortunato 13] S. Fortunato. Benchmark graphs to test community detection algorithms,

https://sites.google.com/site/santofortunato/inthepress2.

[Foster 01] K. Foster, S. Muth, J. Potterat, and R. Rothenberg. A Faster Katz Status Score Algorithm. Computational and Mathematical Organization Theory, Vol. 7, No. 4, pp. 275-285, Dec. (2001)

[Freeman 77] L. C. Freeman. A Set of Measures of Centrality Based on Betweenness. Sociometry, Vol. 40, No. 1, pp. 35-41(1977)

[Girvan 02] M. Girvan and M. E. J. Newman. Community structure in social and biological networks. Proceedings of the National Academy of Sciences of the United States of America, Vol. 99, No. 12, pp. 7821-7826, June (2002)

[Jacomy 11] M. Jacomy. Force atlas 2 layout, http://gephi.org/2011/forceatlas2-the-newversion-of-our-homebrew-layout/, (2011)

[Jarukasemratana 13] S. Jarukasemratana, T. Murata, and X. Liu. Community detection algorithm based on centrality and node distance in scale-free networks. In Proceedings of the 24th ACM Conference on Hypertext and Social Media, HT'13, pp. 258-262, Paris, France, (2013) ACM.

[Katz 53] L. Katz. A new status index derived from sociometric analysis. Psychometrika, Vol. 18, No. 1, pp. 39-43, Mar. (1953)

[Klein 93] D. J. Klein and M. Randić. Resistance distance. Journal of Mathematical Chemistry, Vol. 12, No. 1, pp. 81-95, Dec. (1993)

[Krebs 04] V. Krebs. Political book networks, http://www.orgnet.com/divided.html, (2004)

[Lancichinetti 08] A. Lancichinetti, S. Fortunato, and F. Radicchi. Benchmark graphs for testing community detection algorithms. Physical Review E (Statistical, Nonlinear, and Soft Matter Physics), Vol. 78, No. 4 (2008)

[Lancichinetti 09a] A. Lancichineti and S. Fortunato. Community detection algorithms: A comparative analysis. Phys. Rev. E, Vol. 80, Issue 5, pp. 056117, Nov. (2009)

[Lancichinetti 09b] A. Lancichinetti, S. Fortunato, and J. Kertész. Detecting the overlapping and hierarchical community structure in complex networks. New Journal of Physics, Vol. 11, No. 3, Mar. (2009)
[Leskovec 05] J. Leskovec, J. Kleinberg, and C. Faloutsos. Graphs over Time: Densification Laws, Shrinking Diameters and Possible Explanations. In Proceedings of the Eleventh ACM SIGKDD International Conference on Knowledge Discovery in Data Mining, KDD’05, pp. 117-187, New York, NY, USA, (2005)

[Leskovec 07] J. Leskovec, J. Kleinberg, and C. Faloutsos. Graph Evolution: Densification and Shrinking Diameters. ACM Trans. Knowl. Discov. Data, Vol. 1, No. 1, NewYork, NY, USA, (2007)

[Lusseau 03] D. Lusseau, K. Schneider, O. J. Boisseau, P. Haase, E. Slooten, and S. M. Dawson. The bottlenose dolphin community of Doubtful Sound features a large proportion of long-lasting associations. Behavioral Ecology and Sociobiology, Vol. 54, No. 4, pp. 396-405 (2003)

[McAuley 12] J. McAuley and J. Leskovec. Discovering Social Circles in Ego Networks, Oct. (2012).

[Newman 03] M. E. J. Newman and M. Girvan. Finding and evaluating community structure in networks. Physical Review E, Vol. 69, No. 2, pp. 026113, Aug.(2003)

[Qian 07] R. Qian, W. Zhang, and B. Yang. Community detection in scale-free networks based on hypergraph model. In Proceedings of the 2007 Pacific Asia conference on Intelligence and security informatics, PAISI'07, pp. 226-231, Berlin, Heidelberg, (2007) SpringerVerlag.

[Raghavan 07] U. N. Raghavan, R. Albert, and S. Kumara. Near linear time algorithm to detect community structures in large-scale networks. Physical Review E, Vol. 79, No. 3, pp. 036106, Sep.(2007)

[Ripeanu 02] M. Ripeanu, I. Foster, and A. Iamnitchi. Mapping the Gnutella network: Properties of large-scale peer-to-peer systems and implications for system design. IEEE Internet Computing Journal, Vol. 6, pp. 2002, (2002)

[Shen 10] H. W. Shen and X. Q. Cheng. Spectral methods for the detection of network community structure: a comparative analysis. Journal of Statistical Mechanics: Theory and Experiment, Vol. 2010, No. 10, pp. P10020, (2010)

[Xu 07] X. Xu, N. Yuruk, Z. Feng, and T. A. J. Schweiger. Scan: a structural clustering algorithm for networks. In Proceedings of the 13th ACM SIGKDD international conference on Knowledge discovery and data mining, KDD '07, pp. 824-833, New York, NY, USA, (2007) ACM.

[Yang 12] J. Yang and J. Leskovec. Defining and Evaluating Network Communities based on Ground-truth, ArXiv e-prints, May (2012)

[Zachary 77] W. W. Zachary. An Information Flow Model for Conflict and Fission in Small Groups. Journal of Anthropological Research, Vol. 33, No. 4, pp. 452-473 (1977)

\section{〔担当委員 : Shigeaki Sakurai〕}

Received May 8, 2014.

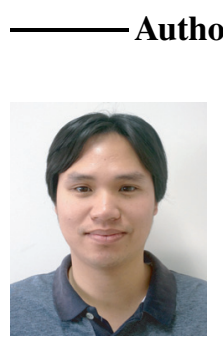

Sorn Jarukasemratana, Dr.

Currently working as a software engineer, he graduated doctural degree from Tokyo Institute of Technology under the supervision of Dr. Tsuyoshi Murata. He graduated his master degree from University of Tampere, Finland with M.Sc. in User Interface Software Development. His bachelor degree is obtained from faculty of engineering, Chulalongkorn University, Thailand with computer engineering major.

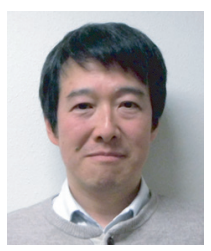

Tsuyoshi Murata, Dr. (Member)

$\mathrm{He}$ is an associate professor in the Department of Computer Science, Graduate School of Information Science and Engineering, Tokyo Institute of Technology. He obtained his doctor's degree in Computer Science at Tokyo Institute of Technology in 1997, on the topic of Machine Discovery of Geometrical Theorems. At Tokyo Institute of Technology, he conducts research on Web mining and social network analysis. 